\title{
Sequential maximum likelihood decoding incorporating reliability determination for image sensor communication
}

\author{
Zhengqiang Tang ${ }^{1, \text { a) }}$, Shintaro Arai ${ }^{1, \text { b) }}$, Tomohiro Yendo ${ }^{2}$, Di He $^{3}$, \\ and Takaya Yamazato ${ }^{4}$ \\ ${ }^{1}$ Okayama University of Science, \\ 1-1 Ridai-cho, Kita-ku, Okayama, 700-0005, Japan \\ ${ }^{2}$ Nagaoka University of Technology, \\ 1603-1 Kamitomioka-machi, Nagaoka 940-2188, Japan \\ ${ }^{3}$ Shanghai Jiao Tong University, 800 Dongchuan Road, Shanghai 200240, China \\ ${ }^{4}$ Nagoya University, Furo-cho, Chikusa-ku, Nagoya 464-8603, Japan \\ a)t19em03tz@ous.jp \\ b)arai@ee.ous.ac.jp
}

Abstract: In this study, the sequential maximum likelihood decoding (S-MLD) for image sensor based visible light communication, referred to as image sensor communication, is proposed. The conventional system cannot correctly decode degraded captured images. To overcome this problem, SMLD partitions the LED area on the image into two segments and sequentially creates pseudo-captured images, which imitate all possible LED blinking patterns. This approach is based on MLD for each segment. Moreover, reliability determination is incorporated to improve communication quality. We performed computer simulations and confirmed that S-MLD exhibits superior decoding performance and approximately $90 \%$ computational cost reduction compared to the previous MLD.

Keywords: visible light communication (VLC), image sensor communication (ISC), maximum likelihood decoding (MLD), sequential maximum likelihood decoding (S-MLD)

Classification: Wireless Communication Technologies

\section{References}

[1] T. Komine and M. Nakagawa, "Fundamental analysis for visible light communication system using LED lights," IEEE Trans. Consum. Electron., vol. 50, no. 1, pp. 100-107, Feb. 2004. DOI: 10.1109/TCE.2004.1277847

[2] T. Yamazato and S. Haruyama, "Image sensor based visible light communication and its application to pose, position, and range estimations," IEICE Trans. Commun., vol. E97-B, no. 9, pp. 1759-1765, Sep. 2014. DOI: 10.1587/ transcom.E97.B.1759

[3] N. Iizuka, "Image sensor communication - current status and future perspectives," IEICE Trans. Commun., vol. E100-B, no. 6, pp. 911-916, June 2017. DOI: 10. 1587/transcom.2016LCI0003

[4] K. Kamakura, "Image sensors meet LEDs," IEICE Trans. Commun., vol. E100-B, no. 6, pp. 917-925, June 2017. DOI: 10.1587/transcom.2016LCI0001

[5] S. Arai, H. Matsushita, Y. Ohira, T. Yendo, D. He, and T. Yamazato, "Maxi- 
mum likelihood decoding based on pseduo-captured image templates for image sensor communication," NOLTA, vol. 10, no. 2, pp. 173-189, April 2019. DOI: $10.1587 /$ nolta. 10.173

\section{Introduction}

Light-emitting diodes (LEDs) represent the latest advancement in the lighting industry. LEDs are gradually replacing traditional lighting implementations because they have high energy efficiency and long life span. Owing to the extensive use of LEDs, visible light communication (VLC) has received significant attention in the optical wireless communication field [1]. This technique transfers data by blinking LEDs at high speed.

The focus of this research is image sensor communication (ISC), which generally refers to the VLC system using a camera as the receiving device [2,3,4]. ISC captures optical signals, which are sent by LEDs, as images and performs image processing to demodulate the data. However, owing to the defocus or pixel loss, the captured images are degraded. Therefore, degraded images limit the communication quality of ISC. A previous study addressed this problem using pseudo-captured image-based maximum likelihood decoding (MLD) [5]. This method creates a pseudo-captured image group corresponding to all possible blinking patterns of the transmitter LED array and extracts one image that is the most similar to the received image from the group as the decoded pattern. Although the optimal demodulation performance of [5] has been confirmed, this approach exponentially increases the computational cost. In this study, we propose sequential maximum likelihood decoding (S-MLD) that achieves communication performance similar to that of MLD and an approximately $90 \%$ computational cost reduction.

\section{System model of ISC}

The block diagram of ISC is shown in Fig. 1(a). The transmitter contains an encoder and an $M \times N$ LED array, and we use on-off keying (OOK) as the modulation method. Binary data is allocated to each LED. We use the blinking of LED to transfer optical signals. Optical signals go through the spatial channel and are captured by the receiver. The receiving device is composed of a camera, an image processing unit, and a decoder. The camera converts degraded optical signals to electrical signals to be output as an image. Then, the image processing unit detects and extracts the LED luminance value as a pixel. Finally, the decoder demodulates data with the extracted pixel according to S-MLD.

\section{Maximum likelihood decoding (MLD) on the basis of pseudo- captured image templates [5]}

Pseudo-captured images are imitations of blinking patterns, which are based on an $M \times N$ LED array transmitter. First, MLD creates all LED blinking patterns, which can be the original received images, as pseudo-captured images. Let $\mathrm{LED}_{m, n}$ be LED in row $m$ and column $n$. Then, the Euclidean distance $D_{p, M, N}$ of pixels between the received image and $p$-th pseudo-captured image is calculated as follows. 


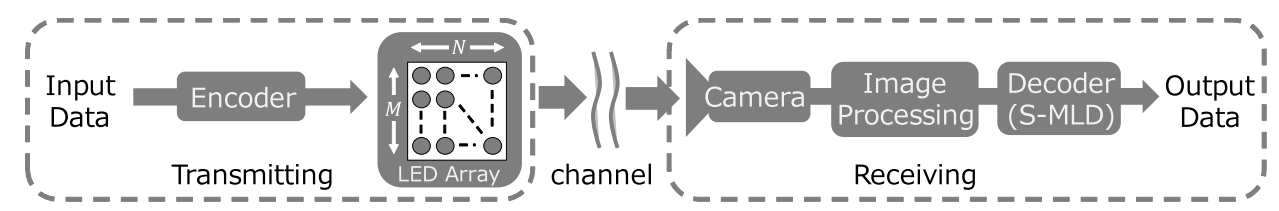

(a) Communication system model of ISC.
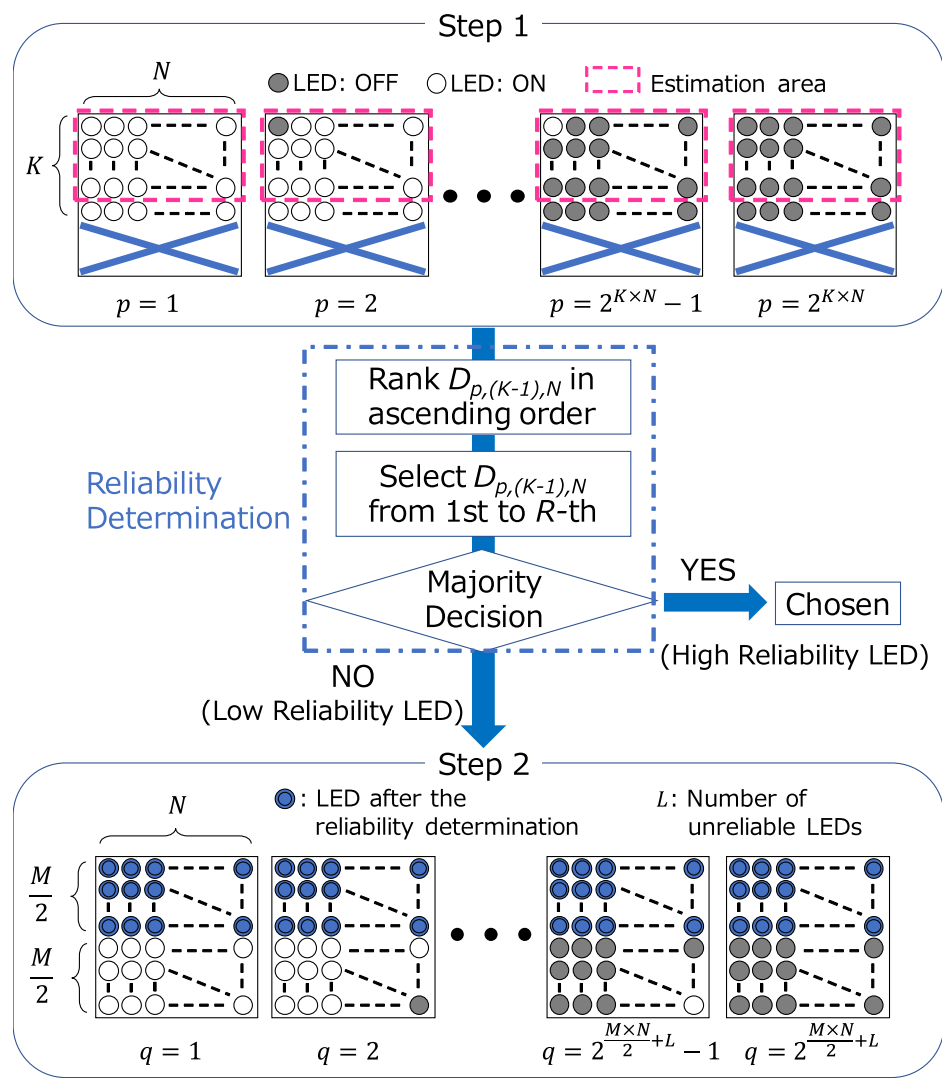

(b) Decoding flow of S-MLD.

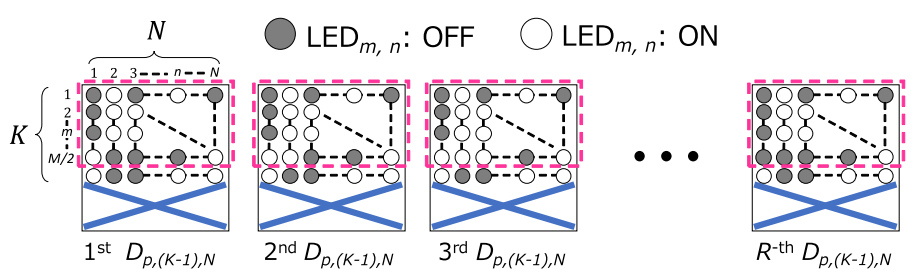

(c) Sample of top $R$ pseudo-captured images.

Fig. 1. Communication system and proposed S-MLD.

$$
D_{p, M, N}=\sqrt{\sum_{m=1}^{M} \sum_{n=1}^{N}\left(R_{m, n}-Z_{p(m, n)}\right)^{2}}
$$

where $R_{m, n}$ and $Z_{p(m, n)}$ represent the $\mathrm{LED}_{m, n}$ pixel value of the received and $p$-th pseudo-captured images, respectively. For MLD, the maximum value of $p$ (i.e., the total number of pseudo-captured images) is $2^{M \times N}$. Finally, a pseudo-captured image with the shortest $D_{p, M, N}$ is extracted, and the data are decoded according to the blinking pattern. It was confirmed that this MLD behavior provides the optimal decoding performance and superior anti-interference ability. However, with an increase in the number of LEDs on the transmitter, MLD requires a considerable computational cost because it must create pseudo-captured images for all LED 
blinking patterns.

\section{Sequential maximum likelihood decoding (S-MLD)}

In this section, we describe a novel S-MLD method that incorporates an LED majority decision to reduce the computational cost of MLD.

\subsection{Demodulation flow}

The demodulation flow of S-MLD is shown in Fig. 1(b). The size of the LED array is set to $M \times N$. In step 1, we first focus on the top $K \times N$ LEDs to create the pseudo-captured images, where $K$ is set to $M / 2+1$. The bottom $(M-K) \times N$ LEDs are excluded to reduce the computational cost. To improve the estimation accuracy, we limit the estimation area to the top $(K-1) \times N$ LEDs and calculate the Euclidean distance $D_{p,(K-1), N}$ for these LEDs. In this case, the maximum value of $p$ is $2^{K \times N}$. Second, S-MLD ranks pseudo-captured images in the ascending order of $D_{p,(K-1), N}$. Then, we select $R$ patterns with the smallest $D_{p,(K-1), N}$ from the first to $R$-th patterns and perform an LED majority decision to determine the reliability. $R$ is the number of candidate patterns required for reliability determination. The details of LED majority decision are presented in Sec. 4.2. After determining reliability, $\mathrm{LED}_{m, n}$ with high reliability is chosen as the transmitted blinking pattern in step 1 . In step 2, we perform MLD, as described in Sec. 3, for the remaining unreliable LEDs together with the bottom $(M / 2 \times N)$ LEDs. The number of unreliable LEDs is indicated by $L\left(0,1, \cdots, L, \cdots, 2^{\frac{M \times N}{2}}\right)$. Then, S-MLD chooses the completed blinking pattern by combining the decoded pattern of step 1 with that of step 2 . Finally, the completed blinking pattern is regarded as the data pattern sent by the transmitter and is used as demodulated data.

\subsection{LED majority decision}

The reliability determination is incorporated after step 1 to avoid demodulation failures. Figure 1(c) shows an example of the proposed LED majority decision. As described in Sec. 4.1, S-MLD extracts the top $R$ blinking patterns in the ascending order of $D_{p,(K-1), N}$. We focus on the top $(M / 2 \times N)$ LEDs in the extracted $R$ patterns and count the ON/OFF status of LEDs at the same position. In this study, we assume that the status of LEDs, which are all ON/OFF, at the same position indicates high reliability, and the data at those positions is decoded as " 1 " or " 0 ". Otherwise, the reliability of decoded LED is determined to be low. LEDs with low reliability are decoded together with the bottom $(M / 2 \times N)$ LEDs in step 2 .

\section{Simulation results}

This section presents computer simulations and describes the performance of the proposed S-MLD compared with that of MLD [5]. The simulation conditions are as follows. In the transmitter, the LED array is composed of $M \times N$ LEDs, and OOK is used as the encoding method. The optical channel matrix, which is based on the $2 \mathrm{D}$ Gaussian filter with a $5 \times 5$ kernel, is defined as follows.

$$
G_{i, j}=\frac{1}{2 \pi \sigma_{g}^{2}} \exp \left(-\frac{i^{2}+j^{2}}{2 \sigma_{g}^{2}}\right) .
$$

where $\sigma_{g}^{2}$ is the variance of the filter, and $i$ and $j$ are, respectively, the vertical and 


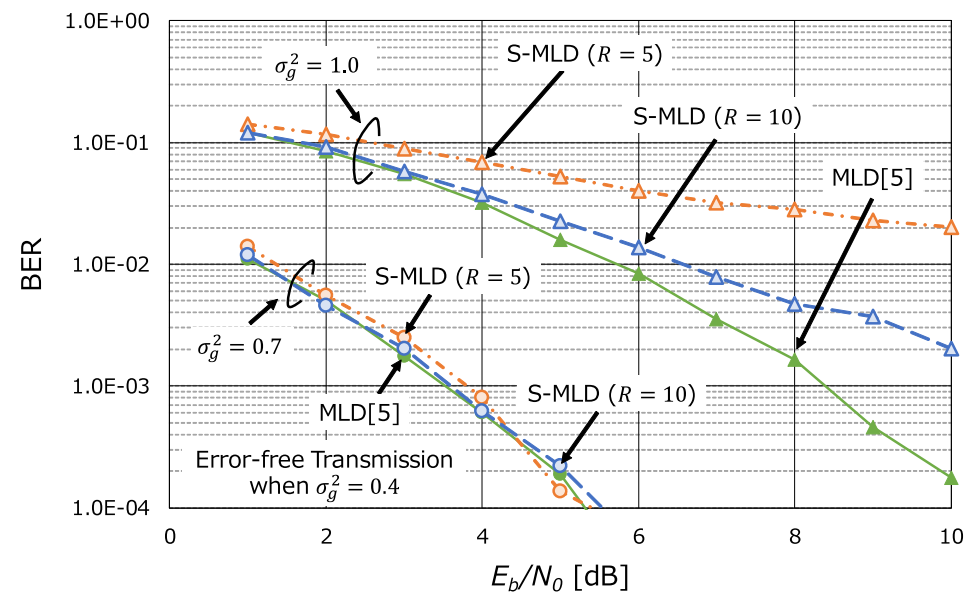

(a) BERs plotted against $E_{b} / N_{0}\left(\sigma_{g}^{2}=0.4,0.7\right.$, and 1.0).

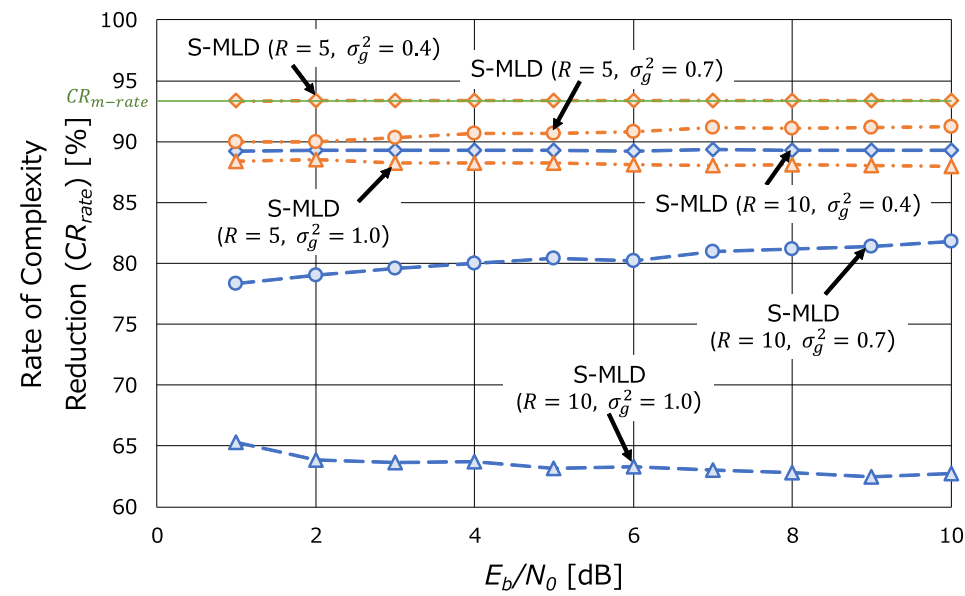

(b) Complexity reduction rate $C R_{\text {rate }}$ versus $E_{b} / N_{0}\left(\sigma_{g}^{2}=0.4,0.7\right.$, and 1.0).

Fig. 2. Simulation evaluation of the proposed S-MLD.

horizontal components of the distance from the origin. After filtering, an additive white Gaussian noise is added to the degraded image as the ambient light noise. During the receiving process, if there is no image degradation in the communication channel, it is assumed that the transmitting LED is captured by one pixel on the received image, and the gap between neighboring LEDs is zero. Therefore, an $M \times N$ LED array is captured by the $M \times N$ pixels without image degradation. The proposed S-MLD performs the LED majority decision to determine reliability. LED majority decision selects top $R$ patterns in the ascending order of $D_{p,(K-1), N}$ and sets the reliability to be high if and only if LEDs at the same position have the same blinking status. In this study, $R$ is set to 5 and 10 . To perform the simulation, $M$ and $N$ are set to a constant value 4 . We fix $\sigma_{g}^{2}$ to $0.4,0.7$, and 1.0 , then change $E_{b} / N_{0}$ in the range of $1-10 \mathrm{~dB}$ to plot BER curves.

Figure 2(a) shows BERs plotted against $E_{b} / N_{0}$ with constant $\sigma_{g}^{2}(=0.4,0.7$, and 1.0). Both MLD and S-MLD achieve error-free transmission under the condition of $\sigma_{g}^{2}=0.4$. When $\sigma_{g}^{2}$ is set to 0.7, the BER curve of S-MLD is similar to that of MLD, indicating that S-MLD achieves an almost equivalent demodulation performance of MLD under this simulation condition. Conversely, if we set $\sigma_{g}^{2}$ to 1.0 , the BER difference between MLD and S-MLD apparently exists. Thus, the captured image 
blur is very strong, and the brightness of all LEDs in the image becomes flat. Then, we assume that the strong blur destroys the feasibility of threshold determination in the OOK transmission. Although the receiver suffers from demodulation of data owing to the strong blur despite the use of MLD, BER performance of S-MLD approaches that of MLD when increasing the value of $R$ to 10. Moreover, BER performance is affected with an increase in the values of $M$ and $N$.

Figure 2(b) shows the complexity reduction rate $C R_{\text {rate }}$ versus $E_{b} / N_{0}$. As described above, MLD creates $2^{M \times N}$ pseudo-captured images to recover data. With an increase in the number of LEDs, the number of template images exponentially increases. Thus, this study uses the total number of calculations of the Euclidean distance to decode the data transmitted as an indicator of the reduction in the computational complexity. The rate of complexity reduction $C R_{\text {rate }}$ is calculated as follows.

$$
C R_{\text {rate }}=\left(1-\frac{T_{\mathrm{S}-\mathrm{MLD}}}{T_{\mathrm{MLD}}}\right) \times 100[\%] .
$$

Where $T_{\mathrm{S}-\mathrm{MLD}}$ and $T_{\mathrm{MLD}}$ are the total number of created pseudo-captured images of S-MLD and MLD, respectively. As we can see, the $C R_{\text {rate }}$ gradually decreases with an increase in the value of $\sigma_{g}^{2}$ because the number of LEDs satisfying the standard of high reliability decreases in a strong blur interference situation. Therefore, more unreliable LEDs are required to be decoded together with bottom LEDs in step 2. When all estimated LEDs in step 1 indicate high reliability, $C R_{\text {rate }}$ achieves its maximum value. The maximum rate of the complexity reduction $C R_{m-\text { rate }}$ is calculated as follows.

$$
C R_{\text {m-rate }}=\left(1-\frac{2^{K \times N}+2^{\frac{M}{2} \times N}}{2^{M \times N}}\right) \times 100[\%] .
$$

The $C R_{m \text {-rate }}$ curve under the condition of $M=N=4$ is plotted in Fig. 2(b). Similarly, the ambient light noise affects $C R_{\text {rate }}$, but not significantly compared with blur interference. Clearly, $C R_{\text {rate }}$ decreases with increasing $R$ because a larger value of $R$ tightens the reliability determination. However, the complexity reduction rate of S-MLD is $87-93 \%$ when $R=5$. Therefore, the proposed method has a significant computational cost reduction performance.

\section{Conclusions}

This study proposed a novel S-MLD that incorporated reliability determination for the image sensor communication. We performed computer simulations and confirmed that S-MLD exhibited superior communication performance that was almost equivalent to that of MLD under the conditions of $\sigma_{g}^{2}=0.4$ or 0.7. Moreover, the proposed S-MLD achieved an approximately $90 \%$ reduction in the complexity cost compared to MLD. In future work, we will perform experiments based on S-MLD for VLC systems.

\section{Acknowledgments}

This work was supported in part by JSPS KAKENHI Grant No. JP17K18282, the joint research program of the Institute of Materials and Systems for Sustainability, Nagoya University, National Natural Science Foundation of China (Grant No. 61971278), and the Equipment Pre Research Foundation of China (Grant No. 61404130218). 